\title{
МЕТОДИКА КВАНТИФИКАЦИИ СОСТОЯНИЯ ТРУДОВЫХ РЕСУРСОВ В КОНТЕКСТЕ УПРАВЛЕНИЯ РАЗВИТИЕМ РЕГИОНАЛЬНОЙ СОЦИАЛЬНО- ЭКОНОМИЧЕСКОЙ СИСТЕМОЙ
}

\author{
(c) 2021 Родионов Дмитрий Григорьевич \\ доктор экономических наук, профессор, Высшая инженерно-экономическая школа \\ Санкт-Петербургский политехнический университет Петра Великого, Россия, Санкт-Петербург \\ E-mail: dmitry.rodionov@spbstu.ru \\ (C) 2021 Карпенко Павел Алексеевич \\ Высшая инженерно-экономическая школа \\ Санкт-Петербургский политехнический университет Петра Великого, Россия, Санкт-Петербург
}

(c) 2021 Конников Евгений Александрович

кандидат экономических наук, доцент, Высшая инженерно-экономическая школа

Санкт-Петербургский политехнический университет Петра Великого, Россия, Санкт-Петербург

E-mail: konnikov.evgeniy@gmail.com

Процесс управления развитием региональной социально-экономической системы требует направленного подхода, вследствие чего система индикаторов состояния региональной социальноэкономической системы предполагает выделение центроида. В рамках данного исследования в качестве центроида конверсии управленческого воздействия в рамках региональной социальноэкономической системы выступает комплексное состояние трудовых ресурсов. Для целей квантификации состояния трудовых ресурсов разработана методика оценки показателей тонального разрыва между информацией, описывающей состояние региональной социально-экономической системы и информацией, описывающей реакцию трудовых ресурсов на состояние региональной социально-экономической системы.

Ключевые слова: региональная социально-экономическая система, трудовые ресурсы, квантификация состояния трудовых ресурсов, тональность, информационная среда, тональный разрыв.

Центроидом конверсии управленческого воздействия выступает комплексное состояние трудовых ресурсов. Данный тезис основан на утверждении о предельной значимости и как атрибутивной, так и акцидентной волатильности трудовых ресурсов. Таким образом, для целей текущей оценки и прогнозирования результатов конверсии управленческого воздействия необходима система квантификаторов состояния трудовых ресурсов.

Состояние трудовых ресурсов может быть дифференцировано в соответствии с множеством классификационных признаков, однако наиболее целесообразным в рамках данного исследования является выделение профессиональных и эмоциональных характеристик трудовых ресурсов. При этом эмоциональные характеристики также можно разделить на моментные и устойчиво-длящиеся. Посредствам системы квантификаторов профессиональных характеристик трудовых ресурсов, могут быть сформированы суждения относительно уровня образования и трудового опыта представителей анализируемой совокупности. Однако одним из ключевых свойств процесса формирования данных характеристик является системность и длительность, что в свою очередь определяет необходимость учета значимого временного лага конверсии управленческого воздействия, теоретически достигающего нескольких лет. Таким образом, наиболее эмоциональные характеристики являются наиболее эффективными индикаторами состояния трудовых ресурсов.

Не данный момент не разработано универсальной методологии оценки эмоциональных характеристик трудовых ресурсов. Существует значимое количество уникальных инструментов области социологии и социальной психологии, однако они как правило базируются на экспертной оценке, что в значительной мере повышает субъективность конечного результата. Таким образом, разработки требует ме- 
тодика оценки эмоциональных характеристик трудовых ресурсов, эффективно отражающих состояние трудовых ресурсов. Базисным этапом данной разработки является определение источника первичной информации. Ключевыми свойствами первичной информации в рамках данного анализа являются объективность, актуальность и универсальность. Рассмотрим последовательно каждое из выделенных свойств и конкретизируем специфику его проявления для существующих дифференцированных источников первичной информации.

Под объективностью первичной информации в рамках данного исследования следует понимать ее независимость от субъекта анализа. Первичная информация должна быть генерируема непосредственно представителями трудовых ресурсов, вне осознания аналитического контекста. Следовательно, в качестве первичной информации может выступать естественная информация, генерируемая непосредственно представителями трудовых ресурсов. Концентратором подобной информации выступают социальные сети, а сама информация представлена как в текстовой, так и в аудиовизуальной форме. Каждый из единиц данного информационного массива не метауровне обладает хронологическим и пространственными характеристиками, которые в свою очередь дают возможность структурирования данных информационных единиц в рамках единого информационного контекста.

Под актуальностью первичной информации следует понимать ее значимость относительно текущего состояния региональной социальноэкономической системы. Исключительно актуальная первичная информация отражает конверсию управленческого воздействия. Данное свойство также максимизируется для естественной цифровой информации, генерируемой в социальных сетях, в первую очередь реактивно по отношению к текущей информационной повестке региональной социально-экономической системы. Таким образом, описанный ранее массив естественной цифровой информации, может быть конкретизирован субмассивом реактивной естественной цифровой информации.

Универсальность первичной информации в первую очередь проявляется в ее всеобъемлющем характере на уровне региональной социально-экономической системы. Необходимость максимизации данного свойства обуслов- лено необходимость предметного абстрагирования, следствием чего усреднение оцениваемых характеристик на уровне всей региональной социально-экономической системы. Данное свойство потенциально максимизируется применительно к реактивной естественной цифровой информации, сформированной по отношению к единому для региона информационному потоку, которым в свою очередь выступает в первую очередь региональная новостная информация. Таким образом, для целей максимизации объективности, актуальности и универсальности информационно-аналитического базиса необходимо структурирование первичной информации в контексте ее формирования и динамической трансформации относительно трансформации единого для региональной социально-экономической системы информационного (в первую очередь новостного) информационного потока. Для достижения поставленной цели наиболее целесообразно в качестве источника первичной информации использовать цифровые концентраторы представителей региональных социально-экономических систем в социальных сетях, основой формируемого информационного потока которых является региональная новостная повестка. Также, необходимым свойством данных концентраторов является возможность потребителей информационных единиц данного формируемого потока генерировать реактивный информационный поток. Таким образом, сопоставление эмоциональных характеристик направленного и реактивного информационных потоков, в рамках исследуемых концентраторов, является ранговой основой для формирования квантификаторов состояния трудовых ресурсов.

Так как наиболее значимая часть единиц как направленного и реактивного информационных потоков сформирована в текстовой форме, математической основой процесса квантификации выступают методы NLP (Natural Language Processing). Для целей оценки эмоциональных характеристик естественной текстовой информации в рамках NLP была развита совокупность инструментов анализа тональности. Тональность - это эмоциональное отношение генератора естественной информации к объекту, выраженное в тексте. Эмоциональная составляющая, выраженная на уровне лексемы или коммуникативного фрагмента. Тональность естественной информационной единицы можно определить 
как функцию лексических тональностей составляющих единиц и правил их сочетания [1]. На прикладном уровне анализ тональности позволяет идентифицировать соотношение эмоциональных характеристик текста. К ключевым эмоциональным (тональным) характеристика естественной информации как правило относят:

- Позитивность. Под уровнем позитивности следует понимать относительный процент присутствия в тексте отдельных лексем, последовательностей лексем и правил их сочетания, указывающих на испытываемые генератором положительные эмоции, являющиеся ответной реакцией на некое событие, объект или явление. Традиционно, психологи к положительным эмоциям относят радость, удовольствие, восторг, ликование, уверенность, гордость, симпатия, доверие, любовь, восхищение, умиление, уважение, облегчение [2].

- Негативность. Под уровнем негативности следует понимать относительный процент присутствия в тексте отдельных лексем, последовательностей лексем и правил их сочетания, указывающих на испытываемые генератором отрицательные эмоции, являющиеся ответной реакцией на некое событие, объект или явление. Данная характеристика является полярной по отношению к позитивности. Традиционно, психологи к негативным эмоциям относят беспокойство, страх, злость, печаль, зависть, отвращение и стыд [3].

- Нейтральность. Под уровнем нейтральности следует понимать относительный процент присутствия в тексте отдельных лексем, последовательностей лексем и правил их сочетания, не указывающих на испытываемые генератором положительные или отрицательные эмоции, являющиеся ответной реакцией на некое событие, объект или явление. Традиционно, психологи к нейтральным эмоциям относят интерес, принятие, удивление, интерес [4].

Приведенные тональные характеристики могут быть названы первичными. Оценка данных параметров применительно к информационному потоку региональной социальноэкономической системы может быть дифференцирована в соответствии с источником генерации информационной единицы на оценку первичных тональных характеристик информации, описывающей состояние региональной социально-экономической системы и оценку первичных тональных характеристик инфор- мации, описывающей реакцию трудовых ресурсов на состояние региональной социальноэкономической системы. Следовательно, совокупность квантификаторов состояния трудовых ресурсов может быть в первую очередь представлена следующими показателями:

$T_{c i}^{\text {neut }}$ - уровень нейтральной тональности информационной единицы i, описывающей состояние региональной социальноэкономической системы.

$T_{c i}^{p o s}$ - уровень позитивной тональности информационной единицы i, описывающей состояние региональной социально-экономической системы.

$T_{c i}^{n e g}$ - уровень негативной тональности информационной единицы i, описывающей состояние региональной социально-экономической системы.

$T_{\text {comi }}^{\text {neut }}$ - уровень нейтральной тональности информационной единицы i, описывающей реакцию трудовых ресурсов на состояние региональной социально-экономической системы.

$T_{\text {comi }}$ - уровень позитивной тональности информационной единицы i, описывающей реакцию трудовых ресурсов на состояние региональной социально-экономической системы.

$T_{\text {coml }}^{\text {neg }}-$ уровень негативной тональности информационной единицы i, описывающей реакцию трудовых ресурсов на состояние региональной социально-экономической системы.

Предоставленная совокупность показателей, основанных исключительно на анализе первичной информации не в полной мере может отражать динамические изменения состояния трудовых ресурсов. На метауровне анализа особую значимость приобретают соотношения первичных тональных характеристик. Одним из наиболее значимых относительных параметров выступает эмоциональный разрыв информационной единицы. Данный параметр рассчитывается как отношение уровня позитивной тональности информационной единицы к уровню негативной тональности информационной единицы, и отражает условный уровень дифференциации эмоциональной насыщенности информационной единицы. Значительный уровень данного показателя указывает на тональную нестабильность информационной единицы. Дополнением к данному параметру выступает общий уровень эмоциональности информационной единицы, рассчитываемый как отношение суммы уровня позитивной и негативной тональностей к уров- 
ню нейтральной тональности информационной единицы. Математическая интерпретация данных показателей представлена моделями 1-4.

$$
\begin{aligned}
& T_{c_{i}}^{\text {dis }}=\frac{T_{c_{i}}^{\text {pos }}}{T_{c_{i}}^{\text {neg }}} \\
& T_{c_{i}}^{\text {full }}=\frac{\left(T_{c_{i}}^{\text {pos }}+T_{c_{i}}^{\text {neg }}\right)}{T_{c_{i}}^{\text {neut }}} \\
& T_{\text {com }}^{\text {dis }}=\frac{T_{\text {com }}^{\text {pos }}}{T_{\text {com }}^{\text {neg }}} \\
& T_{\text {com }_{i}}^{\text {full }}=\frac{\left(T_{\text {com }}^{\text {pos }}+T_{\text {com }}^{\text {neg }}\right)}{T_{\text {com }_{i}}^{\text {neut }}}
\end{aligned}
$$

Где:

$T_{c i}^{d i s}$ - уровень тонального разрыва информационной единицы i, описывающей состояние региональной социально-экономической системы.

$T_{\text {comi }}$ - уровень тонального разрыва информационной единицы i, описывающей реакцию трудовых ресурсов на состояние региональной социально-экономической системы.

$T_{c i}^{f u l l}$ - общий уровень эмоциональности информационной единицы i, описывающей состояние региональной социально-экономической системы.

$T_{\text {comi }}^{\text {full }}$ - общий уровень эмоциональности информационной единицы i, описывающей реакцию трудовых ресурсов на состояние региональной социально-экономической системы.

Приведенная совокупность показателей позволяет описать тональный окрас как общего новостного потока, генерируемого в рамках региональной социально-экономической системы, так и реактивного информационного потока, генерируемого непосредственного трудовыми ресурсами. Ключевой характеристикой, отражающей состояние трудовых ресурсов, выступает соотношение данных тональных характеристик, определяющее тональный разрыв. Тональный разрыв характеризует степень дифференциации эмоциональных свойств информации, описывающей состояние региональной социально-экономической системы и информации, описывающей реакцию трудовых ресурсов на состояние региональной социально-экономической системы. Значительное приращение данного параметра указывает на общесистемное ухудшение состояния трудовых ресурсов, и как следствие на потенциальное снижение темпов развития региональной социально-экономической системы. Математическая интерпретация данных показателей представлена моделями 5-9.

$$
\begin{aligned}
& D_{i}^{\text {neut }}=\sqrt{\left(T_{c_{i}}^{\text {neut }}-T_{\text {com }}^{\text {neut }}\right)^{2}} \\
& D_{i}^{\text {pos }}=\sqrt{\left(T_{c_{i}}^{\text {pos }}-T_{\text {com }}^{\text {pos }}\right)^{2}} \\
& D_{i}^{\text {neg }}=\sqrt{\left(T_{c_{i}}^{\text {neg }}-T_{\text {com }}^{\text {neg }}\right)^{2}} \\
& D_{i}^{\text {dis }}=\sqrt{\left(\frac{T_{c_{i}}^{\text {pos }}}{\left.T_{c_{i}}^{\text {neg }}-\frac{T_{\text {com }}^{\text {pos }}}{T_{\text {com }}^{\text {neg }}}\right)^{2}}\right.} \\
& D_{i}^{\text {full }}=\sqrt{\left(\frac{\left(T_{c_{i}}^{\text {pos }}+T_{c_{i}}^{\text {neg }}\right)}{T_{c_{i}}^{\text {neut }}}-\frac{\left(T_{\text {com }}^{\text {pos }}+T_{\text {com }}^{\text {neg }}\right)}{T_{\text {coum }}^{\text {neut }}}\right)^{2}}
\end{aligned}
$$

Где:

$D_{i}^{\text {neut }}$ - разрыв уровня нейтральной тональности информационной единицы i, описывающей состояние региональной социальноэкономической системы и реактивных по отношению к ней информационных единиц.

$D_{l}^{p o s}-$ разрыв уровня позитивной тональности информационной единицы i, описывающей состояние региональной социальноэкономической системы и реактивных по отношению к ней информационных единиц.

$D_{i}^{\text {neg }}$ - разрыв уровня негативной тональности информационной единицы i, описывающей состояние региональной социальноэкономической системы и реактивных по отношению к ней информационных единиц.

$D_{i}^{d i s}$ - разрыв уровня тонального разрыва информационной единицы i, описывающей состояние региональной социально-экономической системы и реактивных по отношению к ней информационных единиц.

$D_{i}^{\text {full }}$ - разрыв общего уровня эмоциональности информационной единицы i, описывающей состояние региональной социально- 
экономической системы и реактивных по отношению к ней информационных единиц.

Методика оценки приведённых показателей представлен на рисунке 1.

Автоматизация приведенной методики может быть реализована средствами языка программирования Python. Данный выбор обусловлен в первую очередь сущностной дифференциацией задач, представленных в методике. Python позволяет агрегировать решение всех приведенных задач в рамках единой программной среды, и значительно оптимизировать процесс универсализации данного решения посредствам комплексов инструментальных библиотек. С программной точки зрения приведенная методика может быть дифференцирована по два принципиальных последовательных этапа - (1) поиск и агрегирования информации; (2) обработка агрегированной информации. Рассмотрим последовательно каждый из выделенных этапов.

На первичном этапе представленной методики производится поиск и агрегирование как информации, описывающей состояние реги-

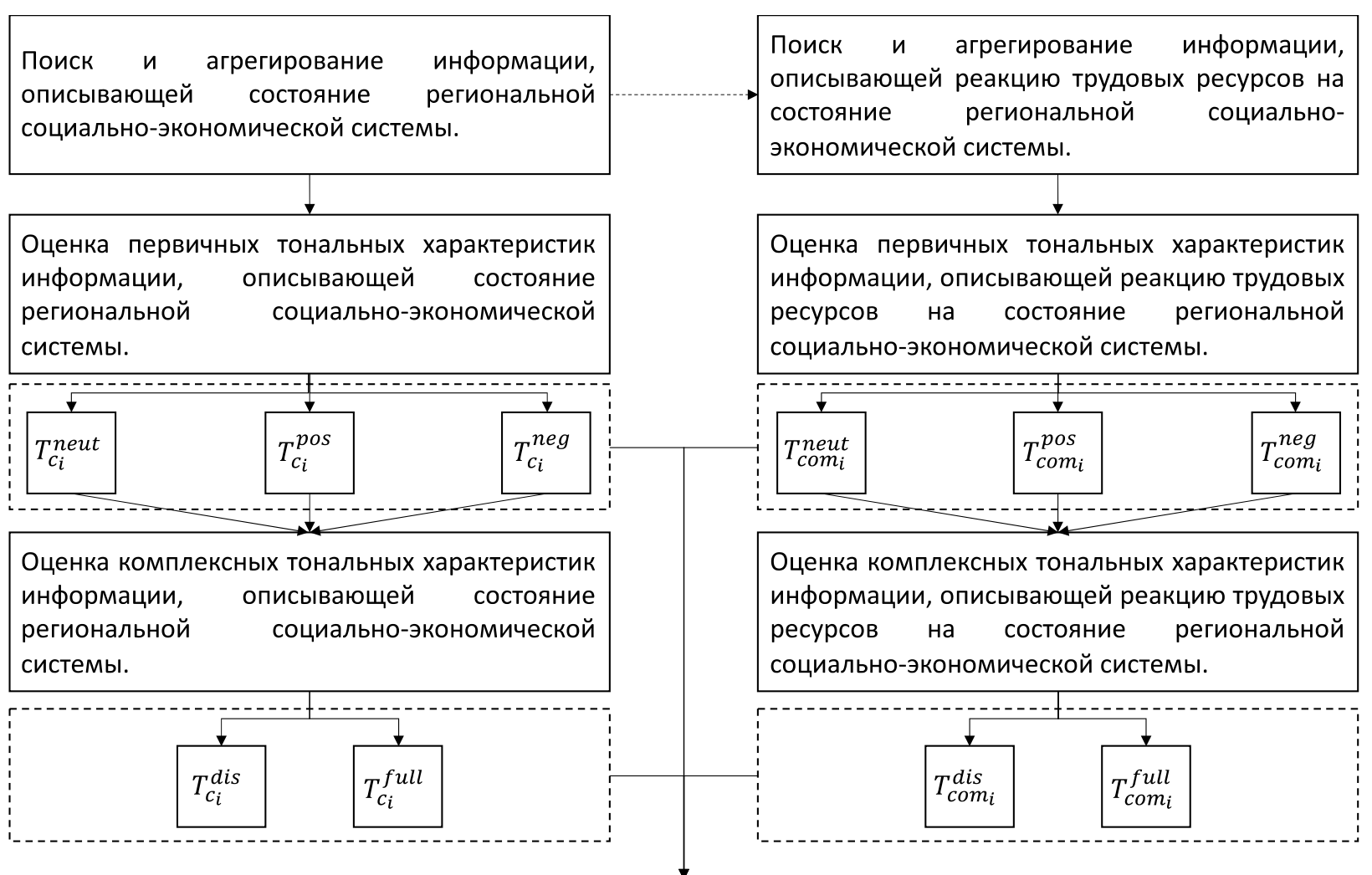

Оценка тонального разрыва между информацией, описывающей состояние региональной социальноэкономической системы и информацией, описывающей реакцию трудовых ресурсов на состояние региональной социально-экономической системы.

\begin{tabular}{|l|l|l|l|}
\hline & \multicolumn{1}{|l}{} & \multicolumn{1}{|c|}{} \\
$D_{i}^{\text {neut }}$ & $D_{i}^{\text {pos }}$ & $D_{i}^{\text {dis }}$ & $D_{i}^{\text {full }}$ \\
\hline
\end{tabular}

Рисунок 1. Методика оценки показателей тонального разрыва между информацией, описывающей состояние региональной социально-экономической системы и информацией, описывающей реакцию трудовых ресурсов на состояние региональной социально-экономической системы 
ональной социально-экономической системы, так и реактивной по отношению к ней информации. В соответствии с выдвинутыми ранее тезисами, наиболее эффективным источником репрезентативной информации выступают информационные концентраторы в социальных сетях, специализированные в области оперативной новостной информации регионального уровня. Несмотря на разнообразие социальных сетей, в рамках реализации данного алгоритма наиболее целесообразно использование социальной сети «ВКонтакте». Данный выбор обусловлен в первую очередь широтой охвата населения, в среднем составляющей 90\% на уровне региона. Следствием данного факта стала интеграция новостных ресурсов в данную социальную сеть и формирование концентраторов репрезентативной реактивной информации. В качестве объекта исследования, для целей апробации разработанной методики, был выбран город федерального значения Санкт-Петербург. Данный выбор обусловлен значимой информационной активностью цифровых СМИ региона, а также значительным сетевым покрытием населения, что потенциально указывает на значимость конверсии управленческого воздействия. Одним из наиболее динамичных распространённых концентраторов новостной информации региональной социально-экономической системы в случае Санкт-Петербурга выступает сообщество «Вести Санкт-Петербург». Данное сообщество является официально зарегистрированным СМИ, содержит исключительно новостную информацию регионального значения, а также концентрирует наиболее коммуникативно активную аудиторию, что определяет значимым объемом реактивного контента. Алгоритм поиска и агрегирования необходимо массива первичной информации подразумевает последовательное извлечение и сохранение ID новостных записей, даты размещения записи, текста размещенной записи, а также массива текстов реактивных по отношению к изначальной записей. Извлекаемая информация агреги- руются в рамках единой матицы. Алгоритм поиски и агрегирования первичной информации представлен на рисунке 2.

По результатам реализации представленного алгоритма применительно к избранному сообществу было получено 56 тысяч информационных единиц. Для целей оценки тональности как новостной информации, так и реактивной информации была выбрана инструментальная библиотека Dostoevsky. Данный выбор обусловлен как высокой статистической точностью инструментария, так и возможностью использования данного инструментария применительно у кириллической текстовой информации. По результатам оценки первичных характеристик тональности информационных единиц, производится расчет и агрегирование представленных ранее характеристик тонального разрыва, которые непосредственно характеризуют состояние трудовых ресурсов. Алгоритм определения показателей тонального разрыва представлен на рисунке 3.

Реализация представленного алгоритма позволила сформировать единый датафрейм, содержащий как тональные характеристики первичной информации, так и показатели тонального разрыва. Полученные результаты требуют усреднения для целей поиска и описания регрессионных связей. Усредненная динамика первичных показателей тонального разрыва представлена на рисунке 4 .

Полученные результаты демонстрируют значимый выброс в 2018 году, причиной чего может быть действия региональных властей в соответствии с реакцией населения на повышение пенсионного возраста, выборы президента РФ и иного контекста политического характера. Таким образом, представленные результаты являются формально-логически обоснованными, что указывает на потенциальную эффективность разработанной методики квантификации информационной среды региональной социальноэкономической системы в рамках оценки комплексного состояния трудовых ресурсов. 
1) Установка необходимых инструментальных библиотек:

- requests - совокупность инструментов автоматизированного извлечения данных из сети интернет.

- datetime - инструменты управления форматом отображения временной информации.

- pandas - совокупность инструментов формирования и обработки многомерных массивов данных.

- time - инструменты управления временем выполнения программного кода.

2) Формирование параметров запроса к API информационного ресурса:

- domain - домен информационного концентратора.

- url - адрес-запрос к API информационного ресурса.

- params - совокупность уточняющих параметров запроса.

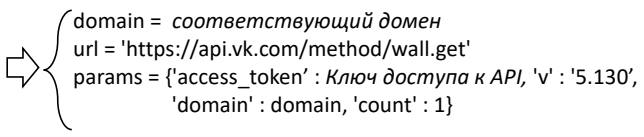

3) Извлечение базовой метоинформации:

- wall_inf 0 - извлеченный массив информации. $\quad$ wall_inf_0 = requests.get(url, params = params)

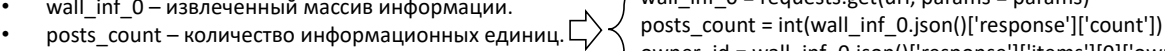

- owner_id - ID информационного концентратора.

4) Извлечение информации описывающей состояние региональной социально-экономической системы:

- offset_iter - список смещений выгрузки информационных единиц.

- all posts inf - совокупный массив извлекаемой информации.
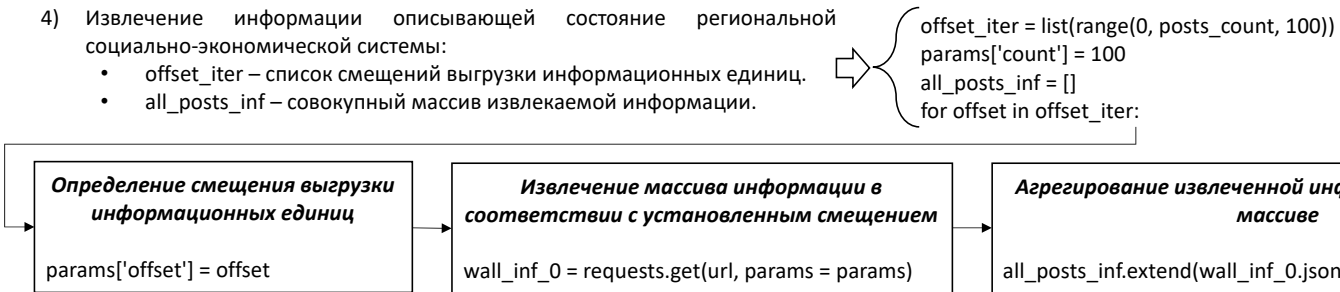

$\rightarrow$\begin{tabular}{c|}
$\begin{array}{c}\text { Извлечение массива информации в } \\
\text { соответствии с установленным смещением } \\
\text { wall_inf_0 = requests.get(url, params = params) }\end{array}$ \\
\cline { 2 - 2 }
\end{tabular}

Агрегирование извлеченной информации в едином массиве

all_posts_inf.extend(wall_inf_0.json()['response']['items'])

5) Структурирование информации описывающей состояние региональной социальноэкономической системы:

- id_list-массив ID информационных единиц.

- date list - массив дат размещения информационных единиц.

- text_list-массив текстов информационных единиц.

- comments_count_list-массив количества реактивных информационных единиц.

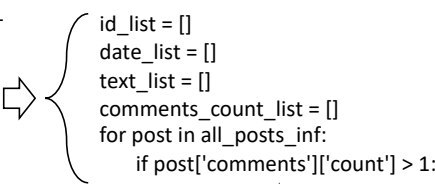

\begin{tabular}{|c|c|c|c|}
\hline $\begin{array}{l}\text { Извлечение и сохранение ID } \\
\text { информационной единицы }\end{array}$ & $\begin{array}{c}\text { Извлечение и сохранение } \\
\text { даты информационной } \\
\text { единицы }\end{array}$ & $\begin{array}{c}\text { Извлечение и сохранение } \\
\text { текста информационной } \\
\text { единицы }\end{array}$ & $\begin{array}{c}\text { Извлечение и сохранение } \\
\text { числа реактивных } \\
\text { информационных единиц }\end{array}$ \\
\hline id_list.append(post['id']) & ftime('\%d-\%m-\%Y')) & text_list.append(post['text']) & post['comments']['count']) \\
\hline
\end{tabular}

6) Извлечение и структурирование информации описывающей (url='https://api.vk.com/method/wall.getComments' реакцию трудовых ресурсов на состояние региональной params['owner_id'] =owner_id социально-экономической системы: comments list - массив текстов реактивных информационных единиц.

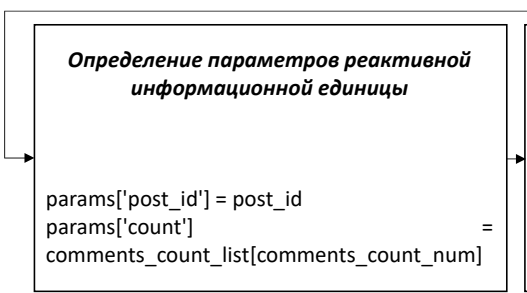

Извлечение данных реактивной информационной
единицы
comment_inf_0 = requests.get(url, params = params)
comments_post_list = []
for comment in comment_inf_0.json()['response']['items']]
if 'text' in list(comment.keys()):
if len(comment['text']) > 2 :
comments_post_list.append(comment['text'])

Агрегирование извлеченной информации в едином массиве

comments_list.append(comments_post_list)

comments count num $+=1$

time.sleep(0.3)

7) Формирование единого датафрейма:

- col_names - наименование столбцов датафрейма.

posts inf df - единый аналитический датафрейм.

(col_names = ['ID', 'Дата', 'Текст записи', 'Число комментариев', 'Комментарии']

$\triangle$ posts_inf_df $=$ pd.DataFrame([id_list, date_list, text_list, comments_count_list, comments_list] $)$

$\checkmark\{$ posts_inf_df $=$ posts_inf_df.T posts_inf_df.columns $=$ col_names

Рисунок 2. Алгоритм поиски и агрегирования первичной информации 
1) Установка необходимых инструментальных библиотек и настройка базовых инструментов:

- pandas - совокупность инструментов формирования и обработки многомерных массивов данных.

- dostoevsky.tokenization - нейронная сеть определения тональных характеристик текста.

- $\quad$ numpy- совокупность инструментов обработки векторов.

\author{
(import pandas as pd \\ from dostoevsky.tokenization import RegexTokenizer \\ $\sqsubseteq\left\{\begin{array}{l}\text { from dostoevsky.mod } \\ \text { import numpy as np }\end{array}\right.$ \\ tokenizer $=$ RegexTokenizer( $)$ \\ model $=$ FastTextSocial NetworkModel(tokenizer=tokenizer)
}

2) Оценка первичных и комплексных тональных характеристик информации, описывающей состояние региональной социально-экономической системы:

- negative_level_list - массив оценок уровня негативности информационных единиц.

- positive_level_list - массив оценок уровня позитивности информационных единиц.

- neutral level list - массив оценок уровня нейтральности информационных единиц.

- tonal disp list - массив оценок уровня тональной дисперсии информационных единиц.

- tonal_full_list - массив оценок уровня комплексной тональности информационных единиц.

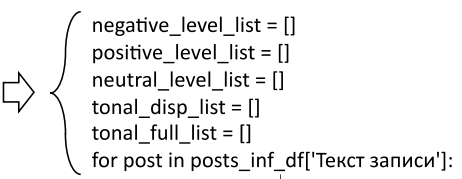

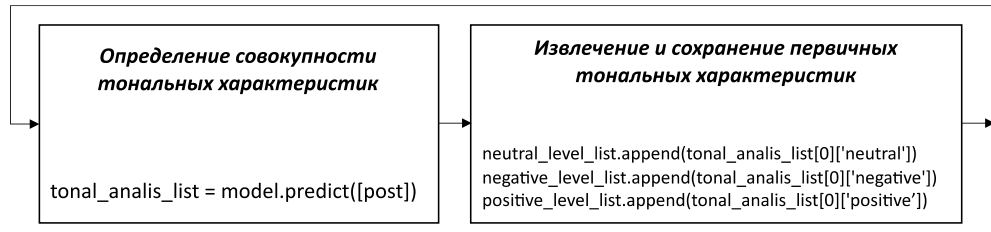

3) Оценка первичных и комплексных тональных характеристик информации, описывающей реакцию трудовых ресурсов на состояние региональной социально-экономической системы:

- negative_level_comments_list - массив оценок уровня негативности реактивных информационных единиц.

positive_level_comments_list - массив оценок уровня позитивности реактивных информационных единиц.

- neutral_level_comments_list - массив оценок уровня нейтральности реактивных информационных единиц.

- tonaldisp comments list - массив оценок уровня тональной дисперсии реактивных информационных единиц.

- tonal_full_comments_list - массив оценок уровня комплексной тональности реактивных информационных единиц.

Определение и сохранение комплексных тональных
характеристик
tonal_disp_list.append(tonal_analis_list[0]['positive']
tonal_analis_list[0]['negative'])
tonal_full_list.append((tonal_analis_list[0]['negative']
tonal_analis_list[0]['positive']) / tonal_analis_list[0]['neutral'])

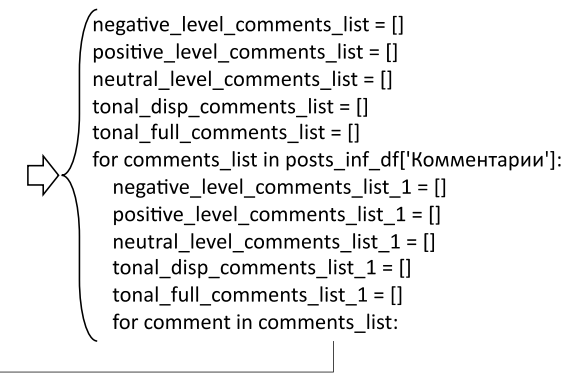

\begin{tabular}{|c|c|}
\hline $\begin{array}{l}\text { Определение совокупности тональных характеристик } \\
\text { tonal analis comment = model.predict([comment]) }\end{array}$ & $\begin{array}{l}\text { Извлечение и сохранение первичных тональных характеристик } \\
\text { neutral_level_comments_list_1.append(tonal_analis_comment[0]['neutral']) } \\
\text { negative_level_comments_list_1.append(tonal_analis_comment[0]['negative']) } \\
\text { positive_level_comments_list_1.append(tonal_analis_comment[0]['positive']) }\end{array}$ \\
\hline
\end{tabular}

Определение и сохранение комплексных тональных характеристик

tonal_disp_comments_list_1.append(tonal_analis_comment[0]['positive'] / tonal_analis_comment[0]['negative'])

tonal_full_comments_list_1.append((tonal_analis_comment[0]['positive'] + tonal_analis_comment[0]['negative']) / tonal_analis_comment[0]['neutral'])

\section{Усреднение тональных характеристи}

negative_level_comments_list.append(sum(negative_level_comments_list_1) / len(negative level comments list 1))

positive_level_comments_list.append(sum(positive_level_comments_list_1) / len(positive_level_comments_list_1))

neutral_level_comments_list.append(sum(neutral_level_comments_list_1) , len(neutral_level_comments_list_1))

tonal_disp_comments_list.append((sum(tonal_disp_comments_list_1)

len(tonal_disp_comments_list_1)))

tonal_full_comments_list.append((sum(tonal_full_comments_list_1) len(tonal_full_comments_list_1)))

4) Оценка тонального разрыва:

- neutral disp-массив оценок тонального разрыва негативности реактивных информационных единиц.

- negative_disp-массив оценок тонального разрыва позитивности реактивных информационных единиц.

- positive_disp - массив оценок тонального разрыва нейтральности реактивных информационных единиц.

- to tonal disp disp-массив оценок тонального разрыва тональной дисперсии реактивных информационных единиц.

- tonal_full_disp - массив оценок тонального разрыва комплексной тональности реактивных информационных единиц.

(neutral_disp $=[]$ negative_disp $=[]$ positive_disp = []

$>$ tonal_disp_disp $=[0$ tonal_full_disp = []

counter_list $=$ list $($ range $(0$ len(posts_inf_df)))

neutral_dist_coef $=(($ neutral_level_list[counter] - neutral_level_comments_list[counter] $) * * 2) * *(1 / 2)$ negative_dist_coef $=(($ negative_level_list[counter] - negative_level_comments_list[counter] $) * * 2) * *(1 / 2)$

positive dist coef $=(($ positive level_list[counter] - positive level_comments_list[counter] $) * * 2) * *(1 / 2)$ tonal_disp_coef $=\left((\text { tonal_disp_list[counter] }- \text { tonal_disp_comments_list[counter] })^{* *} 2\right)^{* *}(1 / 2)$

tonal_full_coef $=(($ tonal_full_list[counter] - tonal_full_comments_list[counter] $) * * 2) * *(1 / 2)$
Агрегирование и сохранение характеристик тонального разрыва

neutral_disp.append(neutral_dist_coef) negative_disp.append(negative_dist_coef) positive_disp.append(positive_dist_coef) tonal disp disp.append(tonal disp coef) tonal_full_disp.append(tonal_full_coef)

\section{Рисунок 3. Алгоритм определения показателей тонального разрыва}




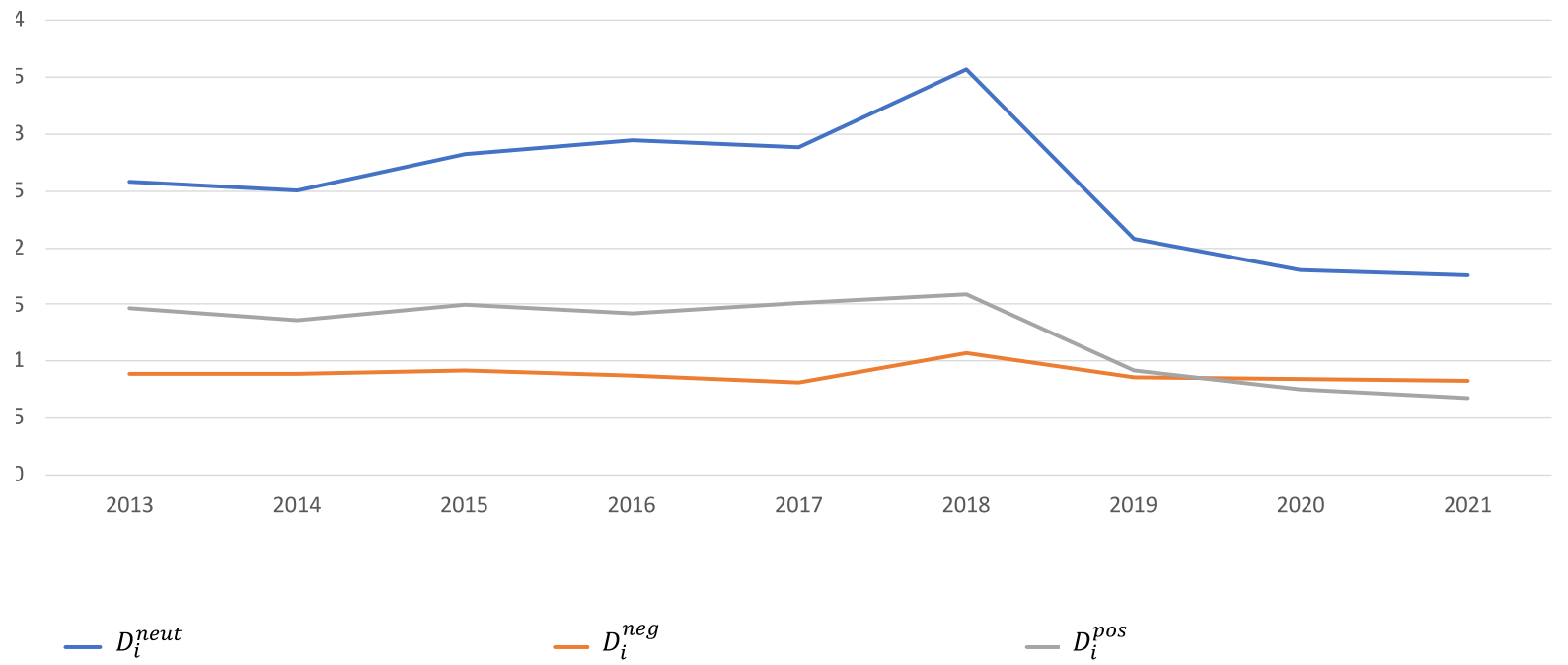

Рисунок 4. Усредненная динамика первичных показателей тонального разрыва

\section{Библиографический список}

1. Bing Liu. Sentiment Analysis and Subjectivity // Handbook of Natural Language Processing (англ.) / под ред. N. Indurkhyа и F. J. Damerau. - 2010.

2. Положительные эмоции.- Текст: электронный // 4BRAIN: [сайт].- URL: https://4brain.ru/blog/ положительный-эмоции/ (дата обращения: 14.04.2021).

3. Негативные эмоции.- Текст: электронный // 4BRAIN: [сайт].- URL: https://4brain.ru/blog/негативныеэмоции/ (дата обращения: 14.04.2021).

4. Путь к эмоциональному интеллекту. - Текст: электронный // СПБГЭУ: [сайт]. - URL: https://unecon.ru/sites/ default/files/emocionalnyy_intellekt.pdf (дата обращения: 14.04.2021).

5. Ершова А.В., Родионов Д.Г., Конников Е.А., Конникова О.А. Системный анализ привлекательности банков для представителей вип-сегмента потребителей в рамках цифровой информационной среды // Экономические науки. 2021. № 194. С. 60-73.

6. Родионов Д.Г., Конников Е.А., Грачева А.А. Анализ системного влияния динамики развития информационных технологий на трансформацию рынка труда // Вестник Воронежского государственного университета. Серия: Системный анализ и информационные технологии. 2020. № 4. С. 64-80.

7. Родионов Д.Г., Конников Е. А., Мугутдинов Р. М. Системный анализ конкурентоспособности цифрового предприятия в рамках информационной среды // Экономические науки. 2020. № 193. С. 394-401.

8. Чан T.T.T., Конников Е.А. Автоматизированная параметрическая модель нормирования бизнеспроцессов на предприятиях деревообрабатывающей промышленности в соответствии с универсальноквантифицированной характеристикой состояния информационной среды // Вестник Алтайской академии экономики и права. 2020. № 12-3. С. 621-637.

9. Конников Е.А., Терентьева Д.А., Конникова О.А. Анализ уровня устойчивого потребления в контексте цифровой информационной среды // Экономические науки. 2020. № 192. С. 114-125. 